\title{
Cholesteryl hemiesters alter lysosome structure and function and induce proinflammatory cytokine production in macrophages
}

\author{
Neuza Domingues a,1 , Luís M.B.B. Estronca ${ }^{\text {b,1 }}$, João Silva ${ }^{\text {b }}$, Marisa R. Encarnação a , Rita Mateus a , Diogo Silva ${ }^{\text {c,d }}$, \\ Inês B. Santarino a , Margarida Saraiva c,d, Maria I.L. Soares ${ }^{\mathrm{e}}$, Teresa M.V.D. Pinho e Melo ${ }^{\mathrm{e}}$, António Jacinto ${ }^{\mathrm{a}}$, \\ Winchil L.C. Vaz ${ }^{\text {a }}$, Otília V. Vieira ${ }^{\mathrm{a}, *}$
}

a CEDOC, NOVA Medical School, Faculdade de Ciências Médicas, Universidade NOVA de Lisboa, 1169-056 Lisboa, Portugal

${ }^{\mathrm{b}} \mathrm{CNC}$ - Center for Neuroscience and Cell Biology, University of Coimbra, Coimbra, Portugal

${ }^{c}$ Life and Health Sciences Research Institute (ICVS), School of Health Sciences, University of Minho, Braga, Portugal

d ICVS/3B's PT Government Associate Laboratory, Braga, Guimarães, Portugal

e CQC, Department of Chemistry, University of Coimbra, 3004-535 Coimbra, Portugal

\section{A R T I C L E I N F O}

\section{Article history:}

Received 17 May 2016

Received in revised form 13 October 2016

Accepted 24 October 2016

Available online 26 October 2016

\section{Keywords:}

Atherosclerosis

Cholesteryl hemiesters

Lysosome malfunction

Inflammation

Oxidized lipids

Zebrafish larvae

\begin{abstract}
A B S T R A C T
Rationale: Cholesteryl hemiesters are oxidation products of polyunsaturated fatty acid esters of cholesterol. Their oxo-ester precursors have been identified as important components of the "core aldehydes" of human atheromata and in oxidized lipoproteins (Ox-LDL). We had previously shown, for the first time, that a single compound of this family, cholesteryl hemisuccinate (ChS), is sufficient to cause irreversible lysosomal lipid accumulation (lipidosis), and is toxic to macrophages. These features, coupled to others such as inflammation, are typically seen in atherosclerosis.

Objective: To obtain insights into the mechanism of cholesteryl hemiester-induced pathological changes in lysosome function and induction of inflammation in vitro and assess their impact in vivo.

Methods and results: We have examined the effects of $\mathrm{ChS}$ on macrophages (murine cell lines and primary cultures) in detail. Specifically, lysosomal morphology, $\mathrm{pH}$, and proteolytic capacity were examined. Exposure of macrophages to sub-toxic $\mathrm{ChS}$ concentrations caused enlargement of the lysosomes, changes in their luminal $\mathrm{pH}$, and accumulation of cargo in them. In primary mouse bone marrow-derived macrophages (BMDM), ChS-exposure increased the secretion of IL-1 $1 \beta$, TNF- $\alpha$ and IL-6. In zebrafish larvae (wild-type AB and PU.1:EGFP), fed with a ChS-enriched diet, we observed lipid accumulation, myeloid cell-infiltration in their vasculature and decrease in larval survival. Under the same conditions the effects of ChS were more profound than the effects of free cholesterol (FC).

Conclusions: Our data strongly suggest that cholesteryl hemiesters are pro-atherogenic lipids able to mimic features of Ox-LDL both in vitro and in vivo.
\end{abstract}

(c) 2016 Elsevier B.V. All rights reserved.

\footnotetext{
Abbreviations: Ox-LDL, oxidized low-density lipoproteins; Nat-LDL, native LDL; BMDM, bone marrow derived macrophages; ChS, cholesteryl hemisuccinate; FC, free cholesterol; LacCer, Lactosylceramide; LCCM, L929-cells conditioned medium; LUVs, large unilamellar vesicles; POPC, 1-palmitoyl-2-oleoyl phosphatidylcholine; PUFA, polyunsaturated fatty acids.

* Corresponding author.

E-mail addresses: neuzadomingues16@gmail.com (N. Domingues), estronca@ci.uc.pt (L.M.B.B. Estronca), joao.silva@nms.unl.pt (J. Silva), marisarego@gmail.com (M.R. Encarnação), Rita.Mateus@unige.ch (R. Mateus), diogosilva@ecsaude.uminho.pt (D. Silva), isantarino@gmail.com (I.B. Santarino), Margarida.Saraiva@ibmc.up.pt (M. Saraiva), misoares@ci.uc.pt (M.I.L. Soares), tmelo@ci.uc.pt (T.M.V.D. Pinho e Melo), antonio.jacinto@nms.unl.pt (A. Jacinto), winchil.vaz@nms.unl.pt (W.L.C. Vaz), otilia.vieira@nms.unl.pt (O.V. Vieira).

${ }^{1}$ Equally contributed.
}

\section{Introduction}

The factors that initiate atherosclerotic lesions and influence their progression are extremely complex and still relatively poorly understood [1]. One of the early distinguishing characteristics of the process, already described by de Duve and Peters in the 1970s [2], is the presence of macrophage-derived foam cells in the arterial wall. They result from ingestion of lipid accumulated in the vascular wall by the macrophages and storage of these lipids in lipid droplets, which leads to the formation of intravascular "fatty streaks". This process is, initially, reversible. However, with time, the internalized lipid cannot be metabolically processed by macrophages, and accumulates in their lysosomal compartment resulting in an irreversible phenotype that has the characteristics of lipidosis manifested in several lipid storage diseases [3,4]. Importantly, the exact molecular mechanisms involved in lysosomal lipid accumulation have not been identified. There is a consensus that 
foam cell formation requires entrapment of low density lipoproteins (LDL) within the arterial intima followed by some modification of the entrapped LDL particles [5]. One modification that LDL particles undergo in the arterial intima is oxidation [6-11]. This modification is important because it allows macrophages to take up these particles through unregulated scavenger receptor pathways [5] or through macropinocytosis [12].

Cholesteryl hemiesters are oxidation products of the core aldehydes present in human atheromata [13] and also in oxidized LDL (Ox-LDL) [14]. So far, although considerable attention has been paid in the literature to the atherogenicity of oxidized phospholipids $[15,16]$ and oxysterols [17] the possible effects of oxidation end products of cholesteryl esters have been largely ignored in the literature. Oxidation of lipids containing polyunsaturated fatty acids is quite complex and results in a plethora of products [16]. One of the end products of oxidation of cholesteryl-esters of polyunsaturated fatty acids is the family of cholesteryl hemiesters [18]. We decided to examine this group of compounds as possible etiological agents of atherogenesis and recently showed [18] that a single compound of this chemical family, cholesteryl hemisuccinate (ChS), when incorporated into native LDL (Nat-LDL) particles and presented to macrophages in vitro was sufficient to mimic two important features of the fate of macrophages exposed to Ox-LDL: 1) Cells exposed to ChS-LDL irreversibly accumulate undigested lipid in enlarged lysosomes; and 2) ChS is cytotoxic and provokes apoptotic cell death.

While the toxicity of Ox-LDL and some of its oxidized lipids to macrophages has been well documented, the expansion and malfunction of the lysosomal compartment in macrophages in the initial stages of atherogenesis have been largely unexplored $[4,19]$. In this work we examine the effects of long-term sub-toxic exposure of macrophages to ChS-LDL (Nat-LDL loaded with ChS), on lysosome morphology and function. Similar to our earlier work on this subject [18], ChS was used as a model of the cholesteryl-hemiester family of compounds that are expected to be produced from polyunsaturated fatty acid esters of cholesterol. The work has been extended to an in vivo model by studying the effects of ChS, presented as part of the diet, on zebrafish larvae.

We show that ChS-LDL, at sub-lethal concentrations, induce formation of enlarged lysosomes in macrophages in vitro. The degradative and exit capacity of endocytic cargo by the lysosomes is inhibited. These outcomes seem to be correlated with accumulation of $\mathrm{ChS}$ in the lysosomal membranes and an increase in lysosomal luminal $\mathrm{pH}$. ChS-LDL also trigger a pro-inflammatory response in mouse primary bone marrow-derived macrophages (BMDM). Finally, by enriching the diet of zebrafish larvae with ChS it is possible to mimic the in vitro phenotypes observed, namely, the intravascular lipid accumulation, the inflammation-induced infiltration of myeloid cells in the caudal vein, and also larval death.

\section{Materials and methods}

\subsection{Chemicals and antibodies}

Cholesteryl hemisuccinate and dextran (mol wt 9000-11,000) were from Sigma. POPC and cholesterol (FC) were from Avanti Polar Lipids (Alabaster, $\mathrm{AL}$ ). $\left[{ }^{3} \mathrm{H}\right]$-Cholesterol was from GE Healthcare. The other chemicals used were of analytical grade from local sources. Bodipy 493/503 (4,4-Difluoro-1,3,5,7,8-Pentamethyl-4-Bora-3a,4a-Diaza-sIndacene), Bodipy FL C5-lactosylceramide (LacCer), Dextran Alexa Fluor ${ }^{\circledR} 546$, BSA-Texas Red ${ }^{\circledR}$, DQ $^{\mathrm{TM}}$ Red BSA and LysoTracker ${ }^{\circledR}$ Red DND-99 were from Molecular Probes. DAPI was from Fluka. The antimouse (ABL-93) Lamp-2 antibody was from the Developmental Studies Hybridoma Bank, (University of Iowa, Iowa City, IA). Filipin and the monoclonal mouse anti-EEA1 antibody were from Sigma. Secondary antibodies were from Molecular Probes or from Jackson Immunoresearch.

\section{2. $L D L$ isolation and ChS incorporation}

Human blood was collected from healthy volunteers at CNC, University of Coimbra and CEDOC, NOVA Medical School. Written informed consent was obtained from all volunteers and approved by the Ethical Review Board of Faculty of Medicine of University of Coimbra and NOVA Medical School. LDL were isolated as described previously [20]. ChS-LDL were prepared by incubating Nat-LDL with ChS-POPC (70:30) $100 \mathrm{~nm}$ diameter unilamellar liposomes as was described in [18].

\subsection{Cell culture}

RAW 264.7 cells (ATCC) were maintained in DMEM and cultivated as described in [18]. BMDM were obtained from femurs and tibias of 8-10 week old C57BL/6 mice from Charles River and all animal procedures were reviewed and approved by DGAV, Portugal. Monocytes were differentiated into BMDM by culturing plated cells for 7 days in DMEM supplemented with 10\% L929-cells conditioned medium (LCCM) in a humidified incubator at $37{ }^{\circ} \mathrm{C}$ under $5 \% \mathrm{CO}_{2}$ atmosphere. The assays were performed with BMDM cultures on day seven.

\subsection{Zebrafish maintenance and feeding}

Zebrafish lines were maintained in accordance with Institutional and National animal care protocols, in a re-circulating system at $28{ }^{\circ} \mathrm{C}$ on a 14-hour-light, 10-hour-dark cycle. Wild-type (AB) and Pu.1:EGFP ( $p U 1:: G a l 4, U A S:: G F P)$ zebrafish embryos were obtained by in vitro fertilization and kept in E3 zebrafish embryo medium at $28{ }^{\circ} \mathrm{C}$ until reaching the desired developmental stage. Zebrafish larvae were fed twice a day during 10 days, starting at the 5 days post-fertilization, with normal diet (from Mucedola, control), FC-enriched (normal food supplemented with $4 \% \mathrm{FC}, \mathrm{w} / \mathrm{w}$, or $52 \mu \mathrm{mol}$ per gram of food), or ChS-enriched diet (normal food supplemented with $5 \% \mathrm{ChS} \mathrm{w/w}$, or $52 \mu \mathrm{mol}$ per gram of food). The food was prepared as described in [21]. To visualize lipid accumulation in the vessels, the food was also supplemented with $10 \mu \mathrm{g} / \mathrm{g}$ of red fluorescent cholesteryl ester (CholEsteryl BODIPY® 542/563 C11, from Molecular Probes).

\subsection{Cell toxicity}

In RAW cells, cell toxicity was measured by the MTT assay as previously described [22]. For BMDM the MTS assay was used as previously reported [23].

\subsection{ChS toxicity to zebrafish larvae}

Zebrafish survival was assessed on the 5th day post-fertilization fed larvae, fed with the different diets for 10 days. Larvae were fed twice a day with different concentrations of FC and ChS. Thirty five larvae were placed in each test chamber for each food condition. Dead larvae were removed daily. Larval death was expressed as a fraction of the total number of individuals subjected to a given condition and compared to a control population subjected to a normal diet.

\subsection{ChS uptake}

After incubating RAW cells with ${ }^{3} \mathrm{H}-\mathrm{ChS}$-LDL or Nat-LDL at $37{ }^{\circ} \mathrm{C}$, cell-associated radioactivity and total cell protein were measured as a function of time in the macrophages.

\subsection{Lysosomal degradation of BSA}

The lysosomal capacity to hydrolyze cargo was assessed by incubating cells, treated for 10 days with $16.5 \mu \mathrm{g} / \mathrm{mL}$ of ChS-LDL or Nat-LDL, with $20 \mu \mathrm{g} / \mathrm{mL}$ of DQ-BSA (stock $1 \mathrm{mg} / \mathrm{mL}$ in PBS) for $3 \mathrm{~h}$ in medium without serum, after which cells were washed and the fluorescence 
emission was followed for $4 \mathrm{~h}$ in a fluorimeter SpectraMax Gemini EM (Molecular Devices) with excitation at $590 \mathrm{~nm}$ and emission at $620 \mathrm{~nm}$. The total cellular protein content was measured by BCA as described below and the results were expressed as relative fluorescence intensity/mg of cell protein.

\subsection{Cargo exit from the lysosomes towards the Golgi}

Cells were treated for 10 days with $16.5 \mu \mathrm{g} / \mathrm{mL}$ of ChS-LDL or Nat-LDL were incubated with BODIPY-LacCer $(2.5 \mu \mathrm{M})$ in DMEM with $1 \%$ FBS for $45 \mathrm{~min}$ and LysoTracker-Red (200 nM) for $15 \mathrm{~min}$ at $37^{\circ} \mathrm{C}$. Cell surface fluorescence of LacCer was removed by washing the cells 3 times with $10 \%$ serum. Cells were followed under a confocal microscope for $3 \mathrm{~h}$ at $37{ }^{\circ} \mathrm{C}$ in RPMI with $10 \mathrm{mM}$ Hepes and $10 \%$ FBS. Z-stack images were obtained for both dyes for co-localization analysis using ImageJ with JACoP plugin.

\subsection{Lysosome $\mathrm{pH}$ measurement}

Lysosomal $\mathrm{pH}$ was measured in cells incubated overnight simultaneously with dextran conjugated with pHrodo and dextran conjugated with Alexa Fluor 647 ( $50 \mu \mathrm{g} / \mathrm{mL}$ of each). The dextran-containing cell culture medium was removed, cells were washed with fresh culture medium and analyzed by fluorescence microscopy after $3 \mathrm{~h}$ in dextran-free medium. The acquisition settings were not changed between different samples. To measure the fluorescence intensity ratio between pHrodo and Alexa Fluor 647 within the lysosomes, the organelles were delimited and the fluorescence intensity was quantified through the ImageJ software. Given that the fluorescence intensity of Alexa Fluor 647 does not change between $\mathrm{pH} 4$ and $\mathrm{pH} 8$ (Supplementary Fig. 2A) whereas that of pHrodo does, and that both probes are internalized to the same extent (Supplementary Fig. 2B), the relative fluorescence intensity of pHrodo measured is an indicator of the lysosomal $\mathrm{pH}$. To verify that Alexa Fluor 647 fluorescence was not affected with $\mathrm{pH}$ its fluorescence was measured in $0.2 \mathrm{M}$ sodium acetate buffer between pH 3.5 and pH 7.5 using a Zenyth 3100 microplate reader from Anthos.

\subsection{Filipin staining and binding experiments}

After incubation of cells with $16.5 \mu \mathrm{g} / \mathrm{mL}$ of ChS-LDL or $16.5 \mu \mathrm{g} / \mathrm{mL}$ Nat-LDL for 10 days, cells were fixed and then incubated with Filipin ( $25 \mu \mathrm{g} / \mathrm{mL}$ in PBS) for $30 \mathrm{~min}$ at RT. The cells were observed under a confocal microscope.

The capacity of Filipin to associate with FC and ChS was evaluated by absorption spectroscopy [24]. A $50 \mu \mathrm{M}$ aqueous solution of Filipin was incubated with different POPC-FC (1:1) and POPC-ChS (1:1) large unilamellar liposomes (LUVs) overnight, at RT. Absorption spectra were obtained using the corresponding Filipin-free lipid suspensions as blanks. Filipin-sterol association curves used the absorbance ratio $\left(\mathrm{Abs}_{320} / \mathrm{Abs}_{356}\right)$ as a function of sterol concentration (FC or ChS) in the system and were fitted with theoretical curves using the Hill equation.

\subsection{Cytokine quantification}

BMDM plated in a 24-well plate were incubated with $200 \mu \mathrm{g} / \mathrm{mL}$ of ChS-LDL or $200 \mu \mathrm{g} / \mathrm{mL}$ Nat-LDL for $24 \mathrm{~h}$. Cells were washed and left unstimulated or stimulated with $20 \mathrm{ng} / \mathrm{mL}$ of LPS during $24 \mathrm{~h}$. After this time point, TNF- $\alpha$, IL-10, IL- $1 \beta$ and IL- 6 secretion were measured by ELISA in supernatant of stimulated macrophages. To calculate the cytokine concentration in the samples, optical density values were compared with a standard concentration calibration curve, using a 4 parameter logistic nonlinear regression model.

\subsection{Staining, image acquisition and analysis}

RAW cells were grown on 24- or 48-well plates and fixed with $4 \%$ PFA for 60 min followed by quenching of the aldehyde groups with glycine or ammonium chloride and permeabilized with methanol for LAMP-2 staining or with Triton X100 for anti-EEA-1 antibody staining. Cells were then incubated with the primary antibodies at RT for $1 \mathrm{~h}$, washed, and finally incubated with the secondary antibodies conjugated with a fluorophore for $1 \mathrm{~h}$. Samples were fixed and analyzed by using a Zeiss Confocal microscope.

For lipid visualization in zebrafish, larvae were euthanized using cooled 4\% PFA and mounted in mounting media prior to imaging with Zeiss LSM710 Confocal microscope. Lipid accumulation was measured by delineating fluorescent deposits area with ImageJ software. For quantification of myeloid cells present in the caudal vein, PU.1:EGFP transgenic zebrafish were used; GFP positive cells were determined from an approximately $9000 \mu \mathrm{m}^{2}$ segment of the vasculature.

For Oil Red-O staining, larvae were fixed with 4\% PFA and rinsed three times with PBS containing 0.5\% Tween-20 (PBS-T) as previous described [25]. Briefly, larvae were stained with Oil Red in 60\% isopropanol, prepared from a stock solution at $0.5 \% \mathrm{w} / \mathrm{v}$ in $100 \%$ isopropanol for $15 \mathrm{~min}$. They were then rinsed twice with $60 \%$ isopropanol, for $5 \mathrm{~min}$ and finally with PBS-T.

\subsection{Western blot}

Cells were treated as described in the figure legends. Cell lysates were resolved by sodium dodecyl sulfate-polyacrylamide gel by electrophoresis (SDS-PAGE), and transferred electrophoretically to polyvinylidene difluoride (PVDF) membranes as previously described [26].

\subsection{Protein assay}

The protein content of cell extracts was measured by the bicinchoninic acid (BCA, Pierce) or by a modified Lowry method (BioRad) assay using bovine serum albumin as a standard.

\subsection{Statistical analysis}

Results are expressed as the mean \pm standard error of the mean (SEM). Statistical significance was assessed by one or two-way ANOVA with a Bonferroni post-test. A $p$ value of 0.05 was considered to be statistically significant.

\section{Results}

\subsection{Long-term exposure to sub-toxic ChS-LDL concentrations caused lysosome enlargement in macrophages}

Atheroma formation in vivo is a very slow pathological process, developing over many years and often decades, initiated by formation of irreversible foam cells from macrophages that invade the vasculature. The acute toxic effects of ChS on macrophages, examined in our previous work [18] include irreversible lipidosis, some enlargement of the lysosomes and apoptotic cell death. However, acute toxicity cannot be the only determinant in the development of atheromata. We, therefore, assessed the effect of long-term exposure of macrophages in vitro to sub-toxic concentrations of $\mathrm{ChS}$ upon lysosomes of the cells. To determine suitable ChS concentrations for our experimental purposes, RAW 264.7 macrophages (hereafter referred to as RAW cells) were exposed to different ChS-LDL concentrations for twenty days as shown in Fig. 1A. Henceforward, any reference to the concentration of "ChS-LDL" implies the $\mathrm{ChS}$ concentration in the cell culture medium, the ChS having been loaded into Nat-LDL particles at a ratio of 1000 molecules of ChS per LDL particle as previously described in detail [18]. LDL was 

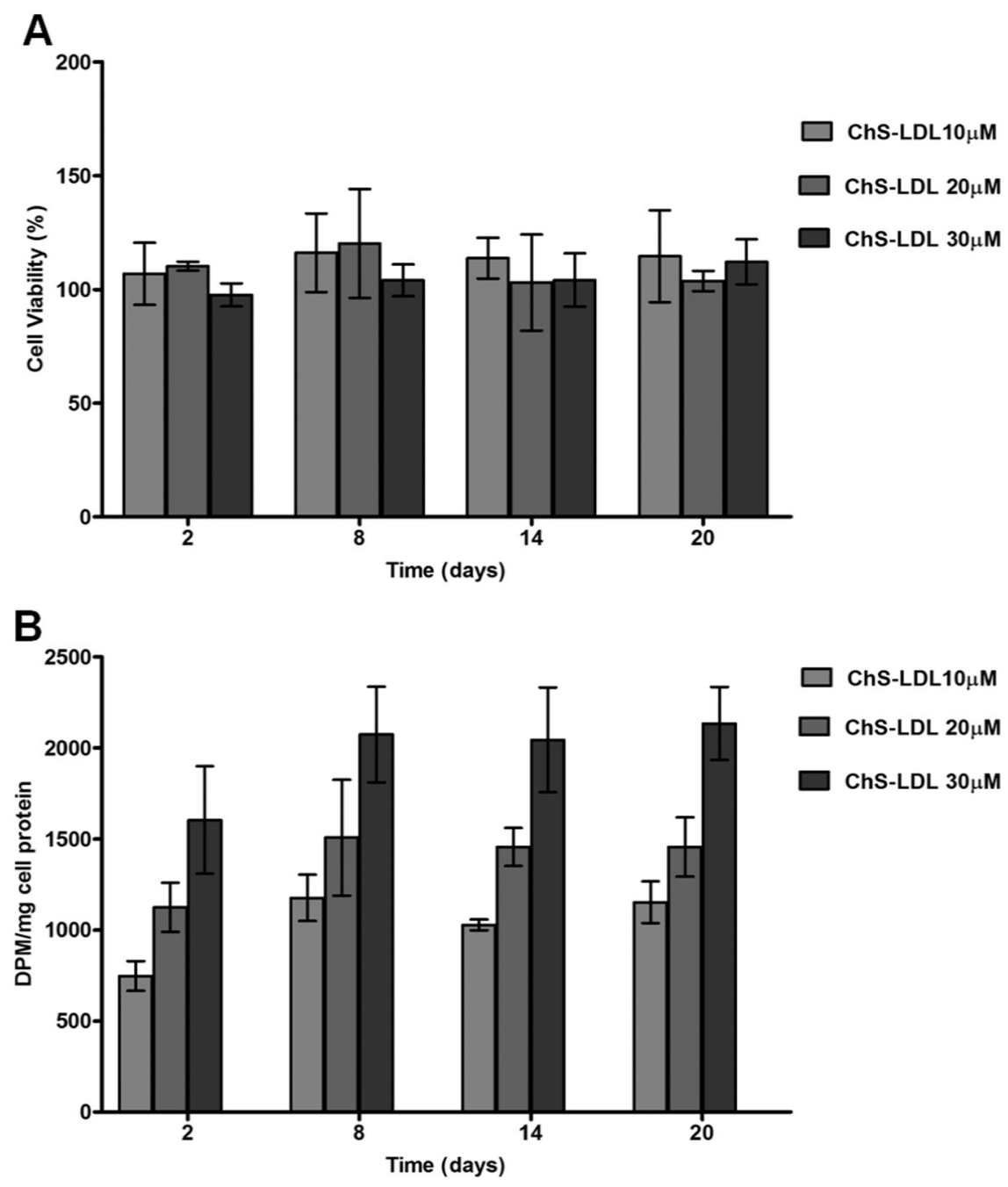

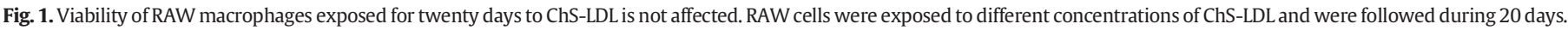

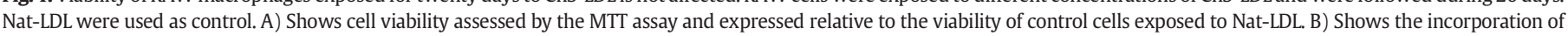

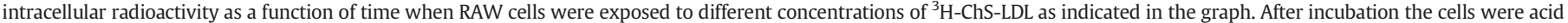
washed and scraped. The radioactivity and protein were quantified as described in the Materials and methods section. Values are mean \pm SEM of three separate experiments.

used as a carrier of the ChS and the ChS/LDL ratio was not supposed to reflect any physiological relevance. Our previous work [18] had shown that acetylated-LDL or phosphatidylcholine liposomes are equally effective carriers for the ChS. The cell cultures were split every two days and, to avoid LDL oxidation due to reactive oxygen species produced by RAW cells, fresh ChS-LDL were added at each splitting. Cell viability, assessed by the MTT assay, was expressed as percentage of the viability of control cells exposed to Nat-LDL that were used in all in vitro experiments described below. Cell proliferation rates were evaluated by counting the cells at each splitting and examining for 5-ethynyl-2'-deoxyuridine incorporation [27] (data not shown). No differences were observed between untreated cells and those treated with Nat-LDL. The three different ChS-LDL concentrations tested were not toxic during the experimental time (Fig. 1A). To ensure that ChS-LDL were being internalized, RAW cells were treated under the same conditions with ${ }^{3} \mathrm{H}-\mathrm{ChS}$-LDL and the intracellular radioactivity was examined as a function of incubation time (Fig. 1B). For all concentrations tested, radioactivity accumulated within the cells in a ChS-LDL concentration-dependent manner and was saturable within the first eight days of incubation (Fig. 1B). We concluded that, at the concentrations tested, ChS-LDL were being internalized by the cells without loss of cell viability.

Taking into account the results described, further experiments used ChS-LDL at a ChS concentration of $30 \mu \mathrm{M}$. RAW cells were exposed to
ChS-LDL for 20 days and lysosomal area and morphology were assessed as a first evaluation of lysosomal function. The first observation was the appearance of large lysosomal structures. The size of the lysosomes was quantified by measuring the area of LAMP-2-positive structures in control and ChS-LDL-treated cells (Fig. 2A). Single $0.419 \mu \mathrm{m}$ thick slices at the maximal cell perimeter were quantified for each case.

Lysosome area in ChS-LDL treated cells ( $n=50$ cells) was strikingly higher than in control cells ( $n=55$ cells) and $~ 40 \%$ of the LAMPpositive organelle area was due to organelles with an area bigger than $6 \mu \mathrm{m}^{2}$ (Fig. 2A, D and E). This feature was not observed in macrophages incubated with Nat-LDL (Fig. 2A-C). Importantly, the number of lysosomes per cell was similar in control and ChS-LDL-treated cells. All LAMP-2-positive structures observed in ChS-LDL were not decorated with EEA-1, a marker for early endosomes (Fig. 2F-G). Thus, the large vacuolar LAMP-2-positive organelles, whose formation was induced by exposure to sub-toxic concentrations of ChS-LDL, were not hybrid organelles of the endocytic compartment. To conclude this set of experiments and to ensure that lysosomal biogenesis was not affected by ChS-LDL treatment we performed a Western blot to assess LAMP-2 protein levels. As shown in Fig. 2H, LAMP-2 levels were similar in cells incubated with Nat-LDL and ChS-LDL. These results suggest that subtoxic concentrations of ChS-LDL induced the formation of enlarged lysosomes in RAW macrophages without changes in their biogenesis. 

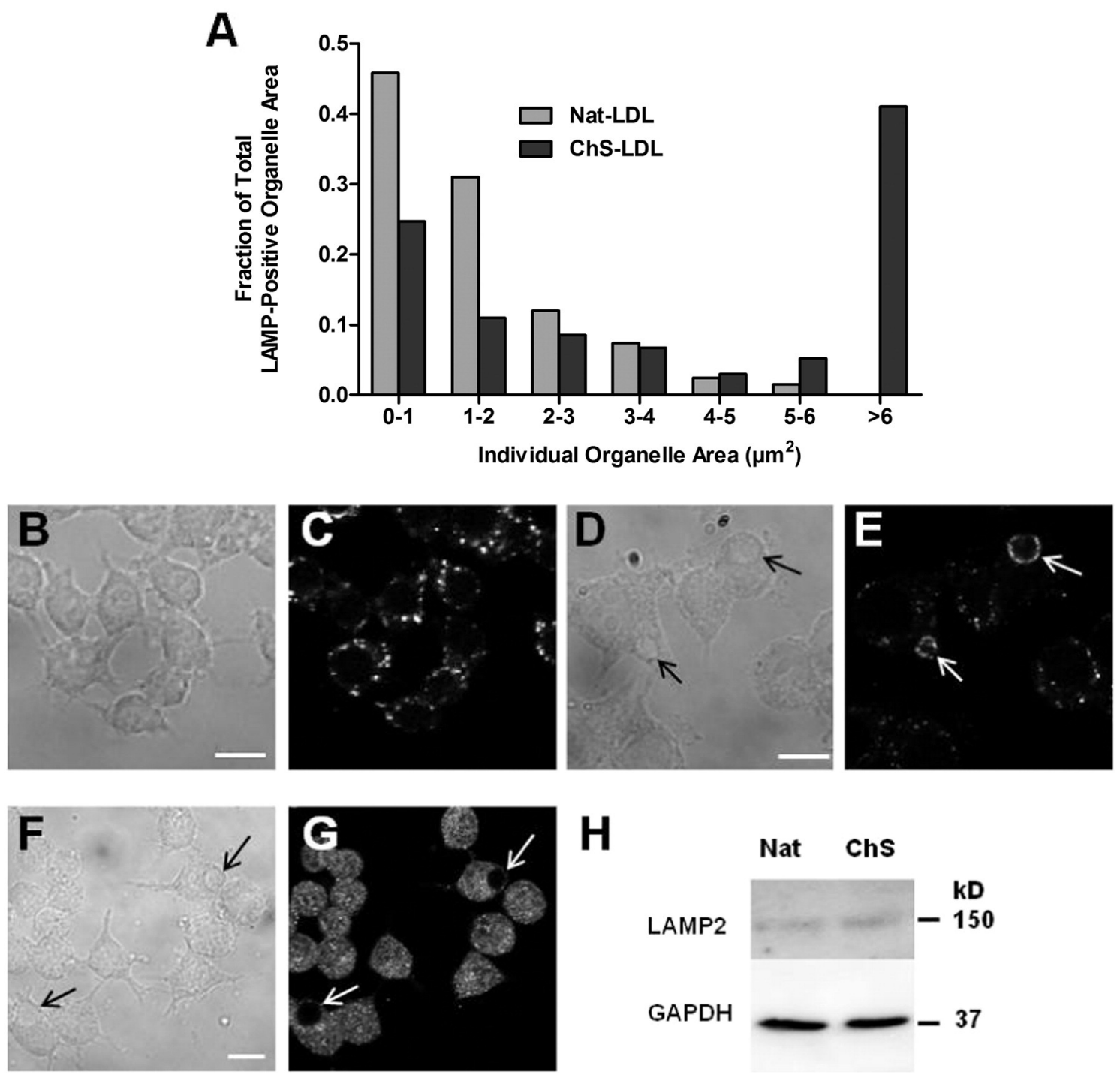

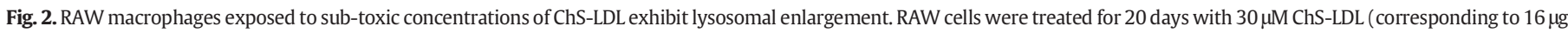

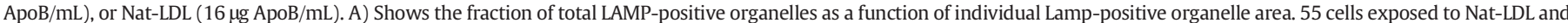

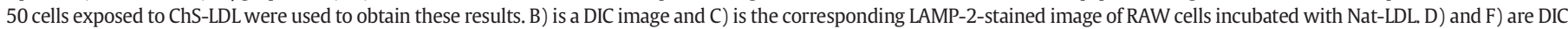

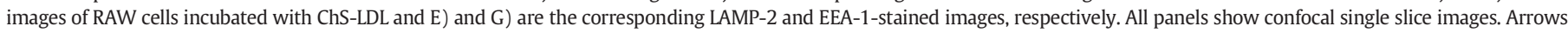
point to the vacuoles. Bars, $10 \mu \mathrm{m}$. H) Shows LAMP-2 protein levels in lysates of cells treated with Nat-LDL or ChS-LDL. GAPDH was used as loading control.

\subsection{Lysosomes in macrophages exposed to ChS-LDL could not degrade} endocytic cargo

Since lysosomes play a central role in receiving and degrading macromolecules from the endocytic membrane-trafficking pathways we examined the effect of ChS in lysosomal degradation of endocytic cargo. Supplementary Fig. 1 shows that neither uptake nor transport of endocytic cargo (BSA conjugated with Texas Red) to the lysosomes were affected by exposure to ChS-LDL when compared to the control (Nat-LDL). Our next step was to verify whether there was efficient degradation of endocytic cargo in the ChS-treated-cells as compared with the control cells. As endocytic cargo we used DQ-BSA, a fluorogenic substrate for lysosomal proteases. Because this cargo is heavily labeled with Bodipy, a strong fluorescence quenching effect is observed. However, upon enzymatic cleavage of the DQ-BSA to dye-labeled peptides in the acidic lysosomal compartments this quenching is relieved, producing brightly fluorescent products. As shown in Fig. 3A-E, the rate of degradation of DQ-BSA by the lysosomal proteases was significantly slower in RAW cells exposed to ChS-LDL as compared to controls, suggesting that the catalytic activity of this organelle was affected.

To assess whether lysosomal exit capacity contributed to lysosomal enlargement in cells treated with ChS-LDL we measured the egress of Lactosylceramide (LacCer) conjugated with Bodipy towards the Golgi. Glycosphingolipids are known to be internalized by endocytosis in a clathrin-independent, caveolae-related mechanism. LacCer, which is not recycled to the PM, is transported to lysosomes and from there to the Golgi apparatus $[28,29]$. RAW cells that had been previously exposed to ChS-LDL were incubated with LacCer and their lysosomes stained with LysoTracker ${ }^{\circledR}$. The kinetics of exit of the LacCer from lysosomes towards the TGN were followed by confocal microscopy in a pulse-chase experiment (Fig. 3F). Similarly to BSA, LacCer delivery to lysosomes did not appear to be impaired in ChS-LDL treated cells since the degree of co-localization of LacCer and LysoTracker® was very similar in control and treated cells at 30 min chase (Fig. 3F). However, with increasing time, co-localization of LacCer and lysosomes decreased in control cells but not in ChS-LDL-treated macrophages. This is clearly 

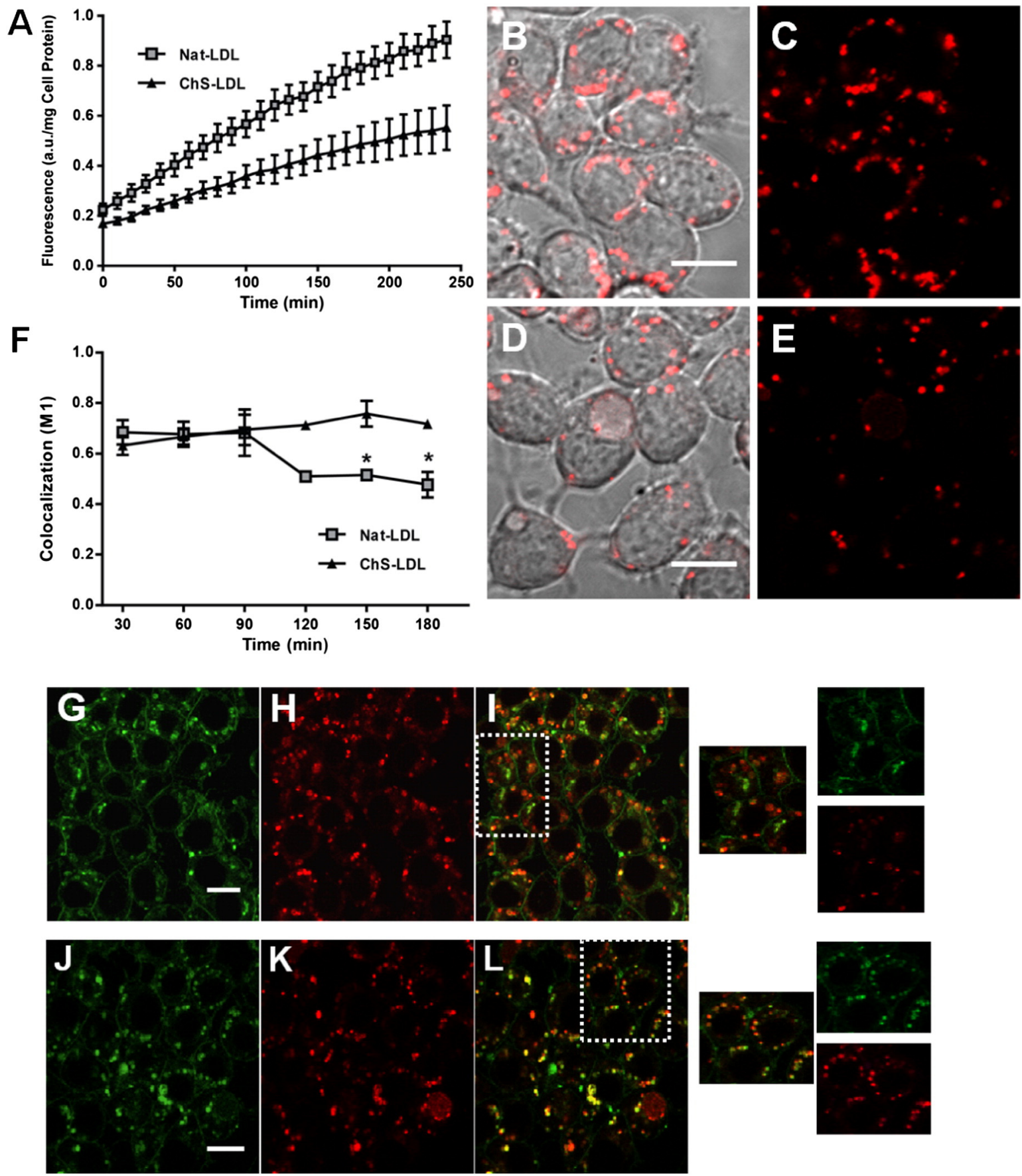

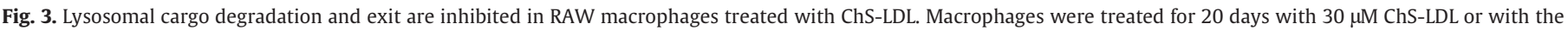

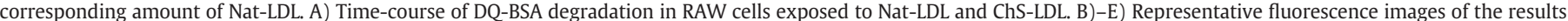

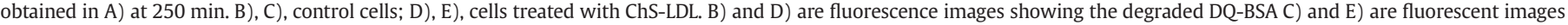

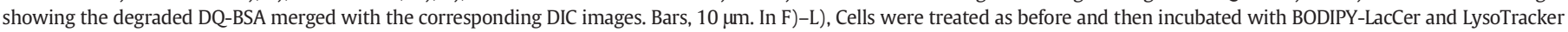

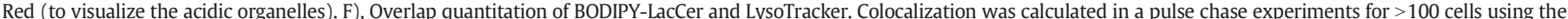

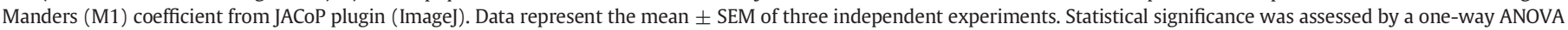

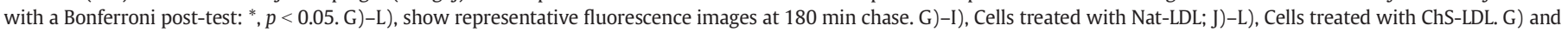
J), BODIPY-LacCer; H) and K), LysoTracker; I) and L) are the corresponding merged images. Bars, $10 \mu \mathrm{m}$. Zoomed regions of the regions outlined in I) and L) are also shown.

seen in the micrographs at 180 min chase (Fig. 3G-L and in the zooms of the regions outlined in panels I and $\mathrm{L}$ ), suggesting that the LacCer exit from lysosomes was inhibited in ChS-LDL treated cells.

We, therefore, conclude that while endocytic cargo was taken up and delivered to the lysosomes at comparable rates in the control and ChS-LDL-treated cells, lysosomal degradation and cargo exit were strongly inhibited in ChS-LDL-treated cells. But why were the lysosomal proteases and cargo exit in ChS-LDL-treated cells affected? To answer this question we measured the lysosomal luminal pH and assessed whether ChS, a non-hydrolysable cholesteryl hemiester [18], was accumulated in lysosomal membranes.

\subsection{ChS-LDL exposure caused alteration of lysosomal $\mathrm{pH}$}

Lysosomal enzymes require an acidic $\mathrm{pH}$ to function properly. Failure to maintain a low pH within the lysosomes would inhibit the lysosomal cargo hydrolysis. We, therefore, measured the lysosomal luminal pH. Cells (Nat-LDL treated controls and ChS-LDL-treated) 
were incubated simultaneously with dextrans conjugated with Alexa Fluor 647 (whose fluorescence is not affected by pH in the range between 4 and 8 ) and with the pH-sensitive pHrodo dye (which has a $\mathrm{pKa} \approx 6.8$ ) (Supplementary Fig. 2A). Like the other endocytic probes used above, the uptake of dextran, a fluid phase marker, was not affected by $\mathrm{ChS}$ (Supplementary Fig. 2B). Changes in $\mathrm{pH}$ were measured by quantifying the ratio of emission at two different wavelengths 584 and $645 \mathrm{~nm}$ for pHrodo and Alexa Fluor 647, respectively. Fig. 4A shows that the fluorescence intensity of pHrodo relative to the fluorescence intensity of Alexa Fluor 647 is decreased in cells treated with ChS-LDL compared with control cells. Considering the expected ratio (for equal internalization of the two fluorescent dextrans) and the $\mathrm{pH}$ titration curves for pHrodo and Alexa Fluor 647 (Supplementary Fig. 2A), a rough estimate of the lysosomal pH in ChS-treated macrophages is $\geq 6$, a significant rise in lysosomal $\mathrm{pH}$ with ChS-LDL treatment. Since optimal lysosome function requires the ability to maintain an acidic $\mathrm{pH}$, typically between 4.5 and 5.0 [30], the inhibition of cargo degradation can be explained, at least in part, by changes in lysosome $\mathrm{pH}$ and subsequent impairment of lysosomal enzyme activity.

We enquired why ChS-LDL exposure could change the lysosomal luminal pH. When RAW cells were exposed to ChS-LDL and stained with Filipin, a dye used to stain FC, the membrane of the enlarged lysosomes were stained brightly (Fig. 4D and E), similar to the plasma membranes of the control and ChS-treated cells (Fig. 4B-E) which are known to contain FC.

Filipin is used as a histochemical stain for FC [24] but there is no report demonstrating that this probe is also able to stain ChS. We showed in Fig. 4F that Filipin associates with $\mathrm{FC}$ and $\mathrm{ChS}$ in liposomes containing these sterols in a similar manner. The experimental data in Fig. 4F was fitted with the Hill equation with dissociation constants for the FilipinFC and Filipin-ChS complexes of 93 and $97 \mu \mathrm{M}$, respectively, and Hill coefficients of 1.7 for both curves. This suggests that both, the association free energy and association stoichiometry, of Filipin with FC and with ChS are identical when these sterols are embedded in phosphatidylcholine bilayers. This result may appear to contradict earlier reports that Filipin associates with FC but not with its esters in bilayer membranes [24,31]. However, it is also possible that the cholesteryl esters used in the earlier work (cholesteryl palmitate and cholesteryl acetate, both neutral cholesteryl esters) were in an isotropic solution state in the bilayer midplane and not in the anisotropic state expected for both FC and $\mathrm{ChS}$ when incorporated into membranes. A more detailed study of the molecular interaction and photophysics of Filipin-sterol complexes in membranes may be justified in the future. Thus, the Filipin staining of the membranes of enlarged lysosomes, combined with the knowledge that $\mathrm{ChS}$ is poorly hydrolyzed in cells [18], suggests the presence of $\mathrm{ChS}$ in them. ChS-induced changes in the biophysical properties of
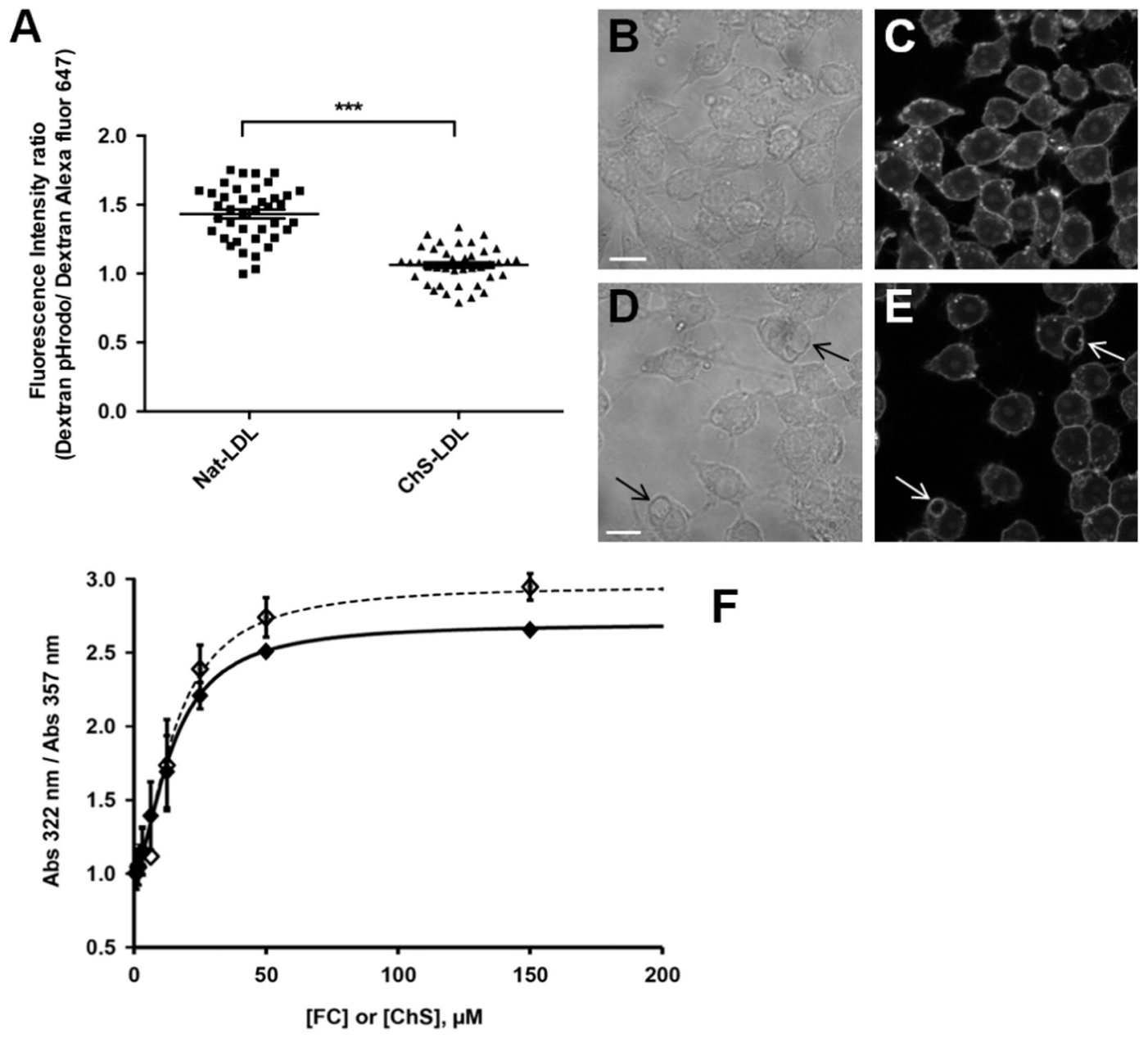

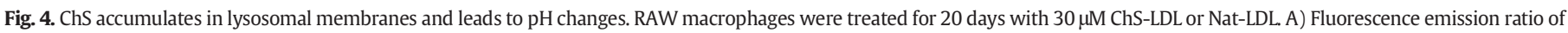

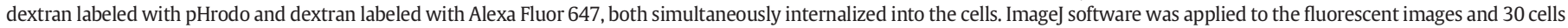

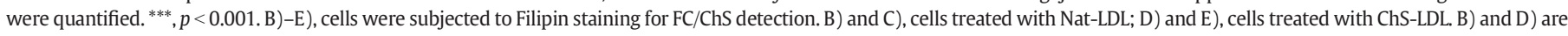

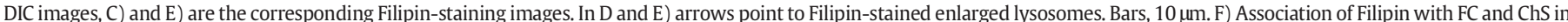

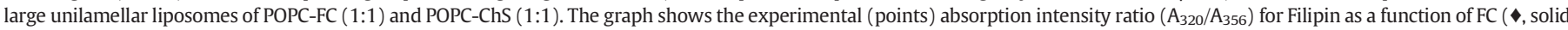

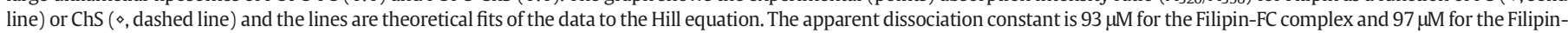
ChS complex, with a Hill coefficient of 1.7 in both cases. 
these membranes could be responsible for the luminal $\mathrm{pH}$ changes which in turn inhibit cargo degradation and possibly its exit.

In light of our data demonstrating the development of lysosomal dysfunction in ChS-LDL-treated macrophages and taking into account that lysosome dysfunction has recently been proposed to result in an increased pro-inflammatory response [32] we assessed whether the ChS-LDL were also able to trigger inflammation, an important feature in atherogenesis.
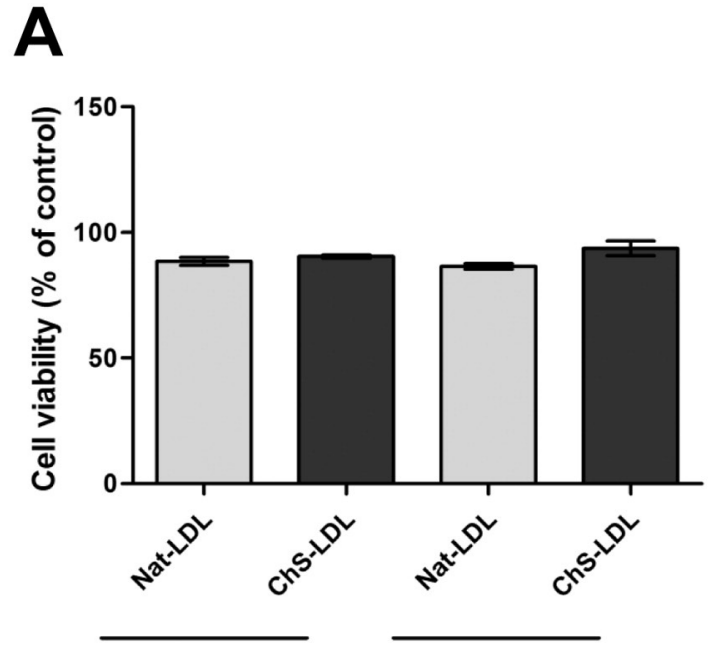

C
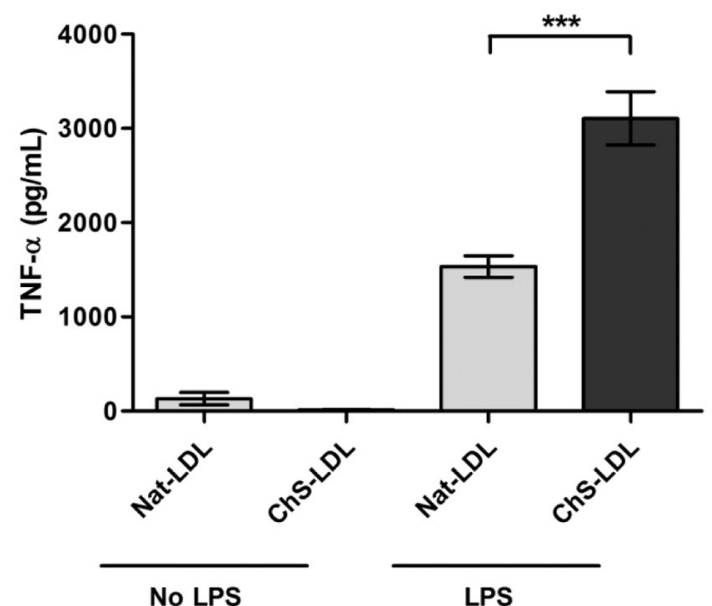

$E$

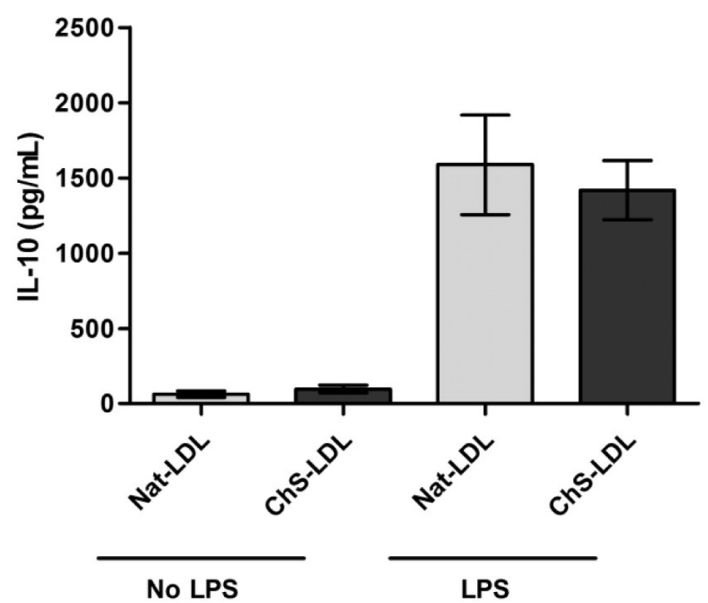

B
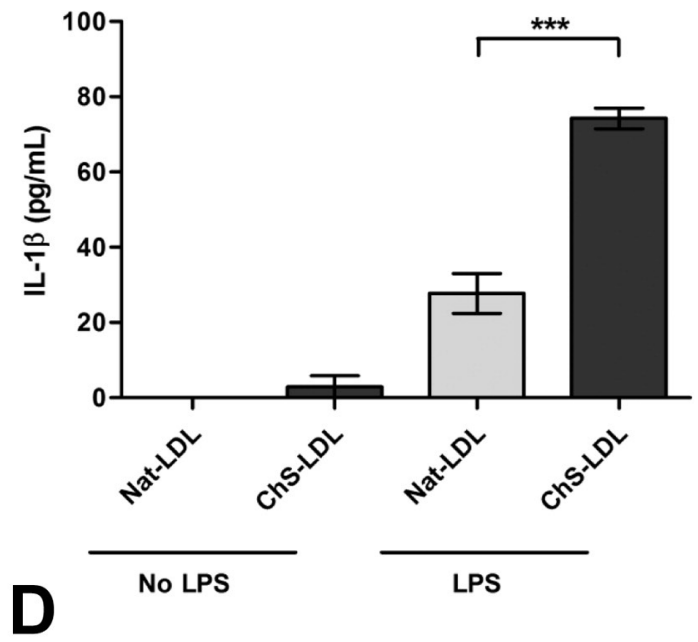

No LPS

LPS

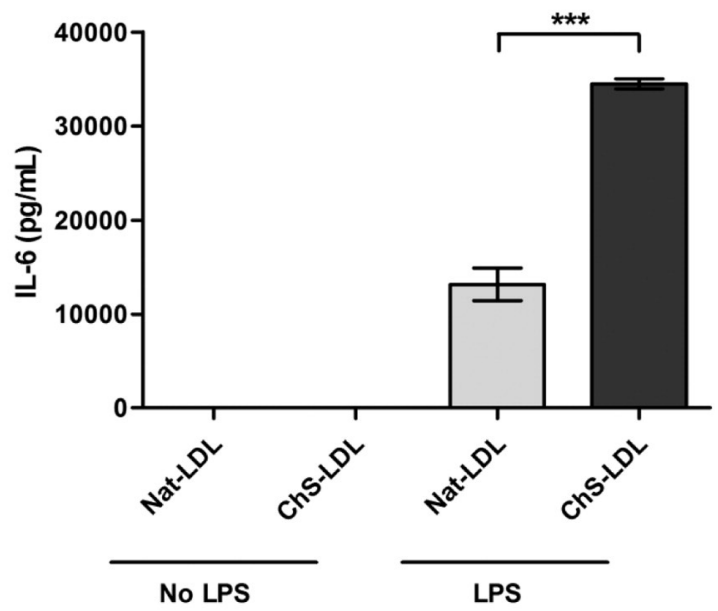

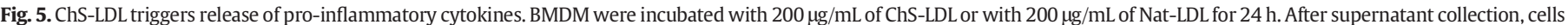

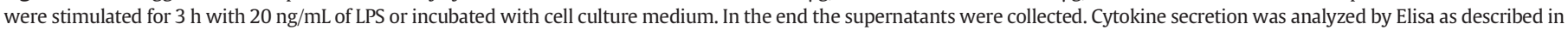

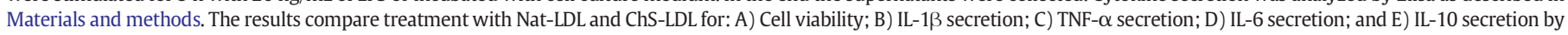
BMDM. ${ }^{* * *}, p<0.001$. 


\subsection{ChS is pro-inflammatory}

We assessed the ability of ChS-LDL exposure to induce inflammatory cytokine secretion by BMDM. Specifically, we determined the levels of IL-1 $\beta$, TNF $\alpha$ and IL- 6 produced by these cells under conditions where cell death did not occur (Fig. 5A). Pre-incubation of BMDM with ChS-LDL for $24 \mathrm{~h}$ and further stimulation with LPS for another $24 \mathrm{~h}$ induced a 2.5 -fold increase of IL-1 $\beta$ release compared to Nat-LDL or to untreated control cells (Fig. 5A). A similar pattern was also observed for TNF- $\alpha$ and IL-6 (Fig. 5B and C, respectively). Statistically different release of the three pro-inflammatory cytokines was always observed between the cells treated with ChS-LDL and Nat-LDL. In contrast, the effect of ChS-LDL on the secretion of the anti-inflammatory molecule IL-10 was not statistically different (Fig. 5D). These results suggest that exposure of the BMDM to ChS-LDL was sufficient to induce an imbalance between pro- and anti-inflammatory cytokines phenocopying what happens in atheroma formation [33-35].

\subsection{In vitro and in vivo ChS effects were positively correlated}

In previous work [18] we showed that exposure of macrophages to high concentrations of ChS-LDL for short periods of time (up to $48 \mathrm{~h}$ ) induced irreversible lipid accumulation in the lysosomal compartment (lipidosis) followed by cell death. Furthermore, these macrophages, that normally hydrolyze cholesteryl esters very efficiently, were neither able to hydrolyze cholesteryl esters nor ChS [18]. In this work we show, for the first time, that $\mathrm{ChS}$ at subtoxic concentrations induces lysosomal malfunction and is highly pro-inflammatory. To further characterize the effects of ChS-LDL in vivo, we decided to pursue our experiments in zebrafish larvae. This in vivo model has recently been shown to be suitable for studying the high-cholesterol diet-induced accumulation of lipids and myeloid cells in the circulatory vasculature walls $[21,36]$.
We started by assessing the percentage of survival of zebrafish larvae fed with a diet enriched in ChS. As controls, we used fish larvae fed with a normal diet (negative control) and a diet enriched in FC (positive control). In all cases larvae were fed for 10 days, starting at 5 days post-fertilization with the same molar concentration of ChS and FC. As shown in Fig. 6A, both FC- and ChS-enriched diets decreased larvae survival in a dose-dependent manner; the ChS-enriched diet being significantly more lethal.

To assess whether ChS was able to induce lipid accumulation in the caudal vein, we used the zebrafish larvae that survived feeding with a $4 \%(\mathrm{w} / \mathrm{w})$ FC-supplemented diet (described in the literature as the amount of dietary FC that is sufficient to induce lipid accumulation in the caudal vasculature [28]), or a $5 \%(\mathrm{w} / \mathrm{w})$ ChS-supplemented diet. (Note that 4\% w/w FC and 5\% w/w ChS correspond to the same number of moles of these compounds in the supplemented diet.) The larval diet was supplemented in all cases with a trace amount of a red fluorescent cholesteryl ester. As shown in Fig. 6B, ChS- and FC-fed larvae showed many focal areas of bright red fluorescence, which we interpreted as lipid accumulation in the vessel wall of the caudal vein. Larvae fed with a normal diet did not exhibit this behavior. The area of the bright red fluorescent structures in the vessel walls, obtained by confocal Z-stacks of the AB zebrafish larvae, was quantified. The area of the lipid deposits in the caudal vein was higher in ChS- than in FC-fed larvae (Fig. 6C). These results are well correlated with the accumulation of lipids induced by ChS-LDL in macrophages [18].

As shown above, ChS-LDL was able to induce secretion of proinflammatory cytokines in primary macrophages. Since one of the hallmarks of inflammation in atherogenesis is the infiltration of myeloid cells into the blood vessels, we assessed leukocyte accumulation in FCand ChS-fed zebrafish vasculature using transgenic PU.1-EGFP zebrafish in which macrophages and granulocytes express GFP. As before, larvae were fed with normal, ChS- or FC-enriched diets for 10 days, starting
A
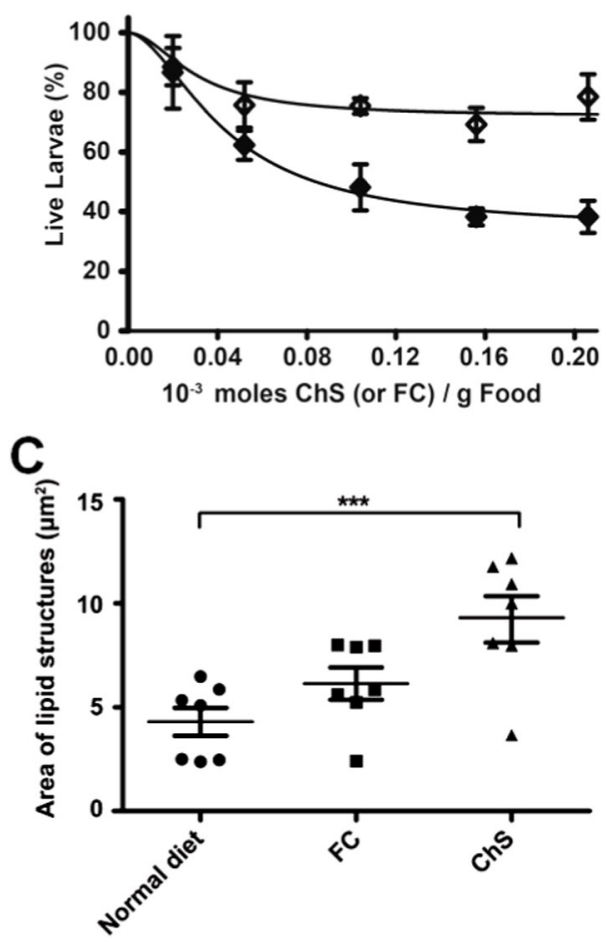

B
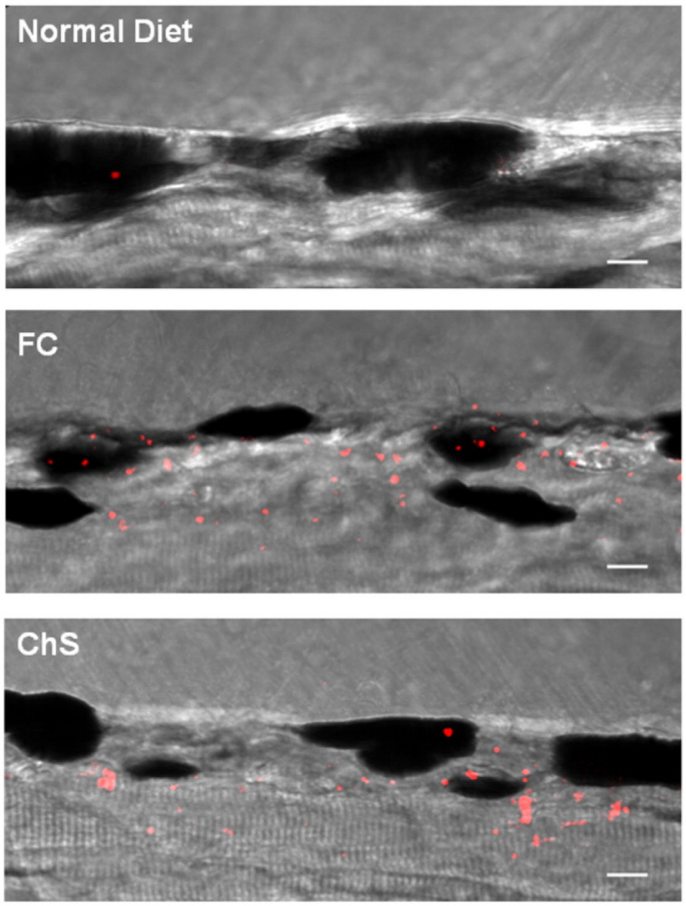

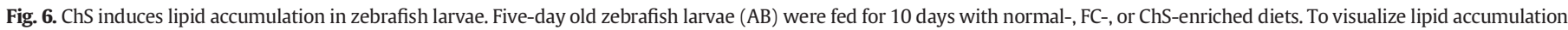

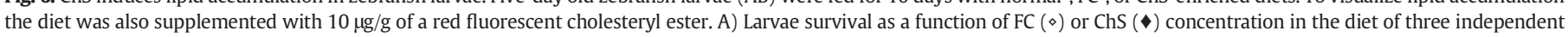

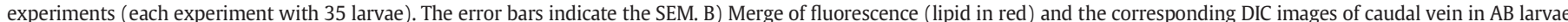

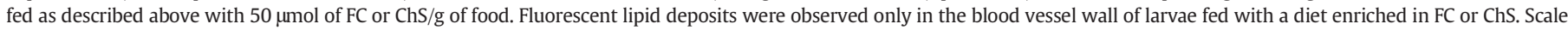

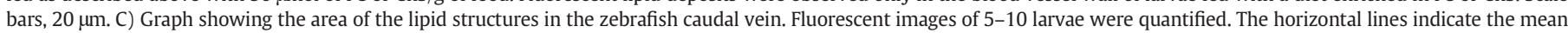
values and the error bars indicate the SEM; ${ }^{* * *}, p<0.001$. 
at 5 days post-fertilization. Feeding with $\mathrm{ChS}$ and $\mathrm{FC}$ resulted in the recruitment of green fluorescent myeloid cells to the caudal vein (Fig. 7), suggesting accumulation of macrophages and/or granulocytes in the vascular wall. Comparison of Fig. 7A and B shows that $\mathrm{ChS}$ induced a higher accumulation of myeloid cells than FC in the vasculature of the transgenic zebrafish larvae. We conclude that $\mathrm{ChS}$ induces inflammation in vivo, a result that is well correlated with the in vitro results (Fig. 5). Additionally, the cells that invade the vasculature are full of neutral lipids, as visualized by confocal microscopy after Oil-Red staining (Fig. 7C).

\section{Discussion}

Physical trapping of LDL particles in the arterial intima followed by their modification is generally understood to be the first step in atherogenesis. In spite of much debate over the past three to four decades as to the exact nature of the modification of the trapped LDL, oxidative modification appears to command a majority consensus. Polyunsaturated fatty acids (PUFA) are among the chemical components of LDL that are most vulnerable to oxidation. In LDL particles these PUFA appear as components of phospholipids and esters of cholesterol. Oxidative scission of PUFA-cholesteryl esters leads to the formation of oxo-esters of cholesterol which have been identified in the "core aldehyde" fraction of ex vivo samples of human atheromata [13] and have long been known to be components of oxidized LDL [14]. As we have argued earlier [18], oxo-esters of cholesterol can be expected to be further oxidized to the corresponding hemiesters of cholesterol. Indeed, these hemiesters of cholesterol are detectable in the plasma of human blood (W. Vaz et al., unpublished results). Work from other laboratories has identified them as the components of Ox-LDL that bind to the plasma protein, $\beta_{2}$-glycoprotein-1, resulting in complexes that may be related to increased acute coronary syndrome risk [37-39].
In previous work [18] we had shown that ChS loaded into native, non-oxidized LDL and presented to macrophages in vitro induced lipidosis in these cells, forming irreversible "foam cells", and leading to dose-dependent cell death. In the present work we have shown that long-term exposure to sub-toxic concentrations of ChS leads to lysosomal malfunction and an inflammatory response in macrophages in vitro. The lysosomal malfunction is characterized by an expansion of the lysosomal compartment, unaffected endocytic cargo uptake and delivery to lysosomes, and a reduction in hydrolytic capacity and exit of cargo from the lysosomes. The demonstrated accumulation of $\mathrm{ChS}$ in lysosomal membranes of macrophages exposed to ChS-LDL probably alters their biophysical properties and, consequently, the activity of V-ATPase, the proton transporter responsible for lysosomal acidification [40]. The resulting increase in lysosomal luminal $\mathrm{pH}$ leads to impairment of cargo degradation and transport across the lysosomal membrane that together culminate in the formation of large lysosomes.

Lysosomal dysfunction is also a critical step in local inflammation in the vessel wall in atherosclerosis [41]. The observation that $\mathrm{ChS}$ also triggers the production of several pro-inflammatory cytokines including IL-1 $\beta$, TNF- $\alpha$ and IL-6, previously described to contribute to formation of atherosclerotic lesions [41,42], correlates well with this information.

Recently, zebrafish have been proposed as an in vivo model for studies on atherogenesis [21,36]. The proteins involved in the transport of dietary fat and lipids and inflammatory pathways in zebrafish are conserved relative to mammals [43-45]. Many of the pro-atherogenic properties of ChS observed in vitro were also observed in vivo in the zebrafish model in which ChS was delivered through the diet. Dietary $\mathrm{ChS}$ was more toxic to the zebrafish than FC at the same molar fraction in the food. Vascular lipid deposition and myeloid cell accumulation in the caudal vein of zebrafish larvae was observed for both FC- and ChS-enriched diets but the results were significantly more pronounced
A
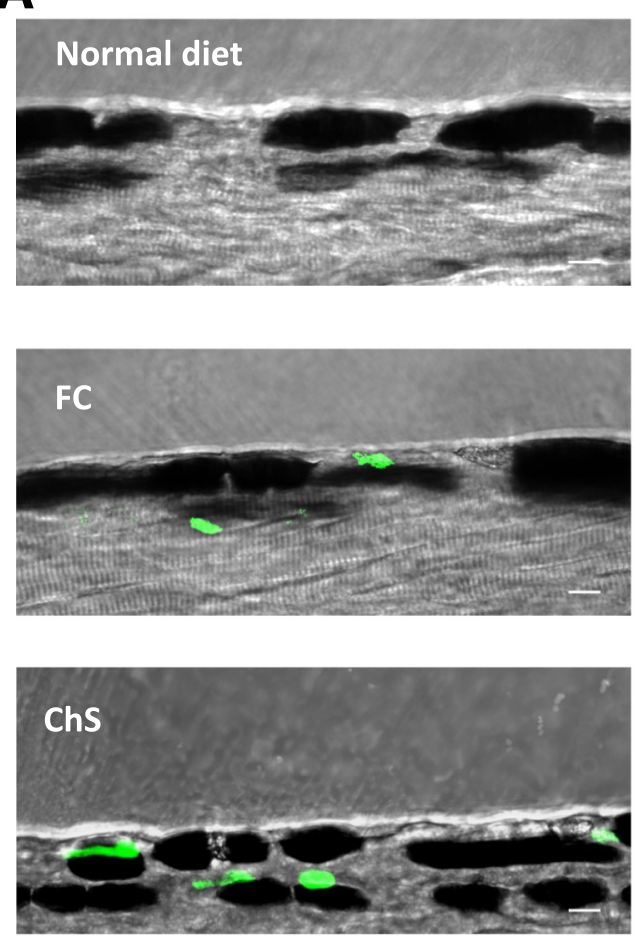

B

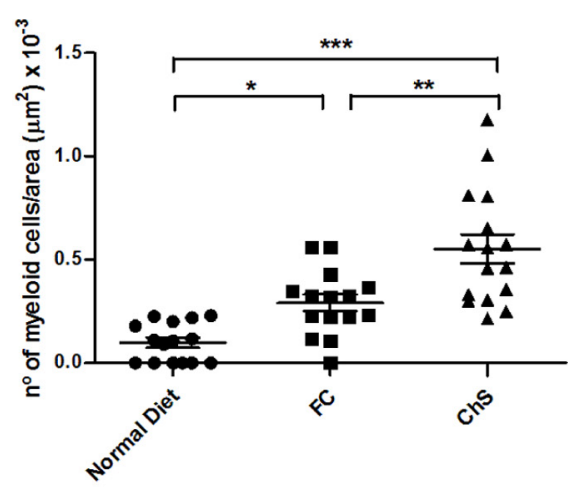

C
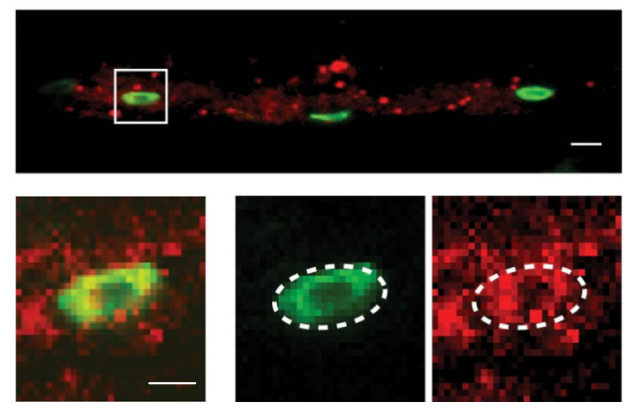

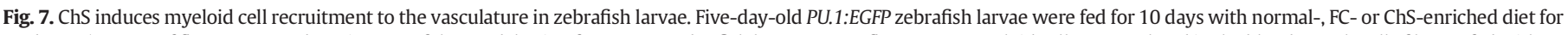

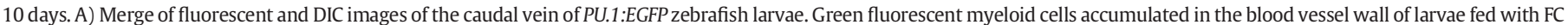

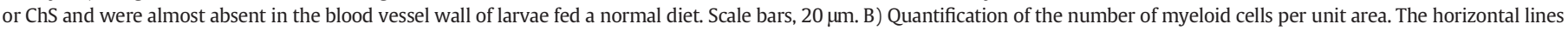

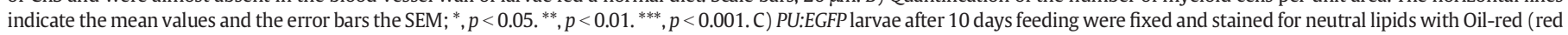
fluorescence). The square in the merged image was zoomed and the green and red channels separated to show a representative myeloid cell full of neutral lipids. Scale bars, 10 m. 
for ChS- than for FC-fed larvae. Hence the in vivo animal model results strongly confirm the in vitro observations using macrophage cell cultures.

\subsection{Conclusions}

Our achievements provide an important conceptual advance vis-à-vis the existing knowledge on two of the salient features of atherosclerosis - lipidosis and inflammation. We have shown, for the first time, that a single product of the oxidation of the low density lipoproteins, ChS, is sufficient to induce both the initiation of atheroma formation (lipidosis) and exacerbate the pathology (inflammation). Simultaneously, we are providing significant new mechanistic insights, showing very clearly that lysosomal malfunction is an important step in the ChS-induced pathogenic process.

Furthermore, one of the guiding motives of our work is the conviction that identification of the chemical etiology of atherogenesis will permit induction of well-defined pathological states in appropriate in vitro or in vivo models in a controlled manner and lead to the subsequent identification of appropriate pharmacological approaches that may identify therapeutic agents.

\section{Conflicts of interest}

We declare no conflicts of interest.

\section{Transparency document}

The Transparency document associated with this article can be found, in the online version.

\section{Acknowledgments/grant support}

This work was supported by iNOVA4Health - UID/Multi/04462/ 2013, a program financially supported by Fundação para a Ciência e Tecnologia (FCT)/Ministério da Educação e Ciência, through national funds and co-funded by FEDER under the PT2020 Partnership Agreement.

FCT fellowship references: SFRH/BPD/26843/2006, SFRH/BD/62126/ 2009, SFRH/BD/90258/2012, SFRH/BD/84685/2012, SFRH/BPD/102229/ 2014, SFRH/BD/52293/2013.

\section{Appendix A. Supplementary data}

Supplementary data to this article can be found online at http://dx. doi.org/10.1016/j.bbalip.2016.10.009.

\section{References}

[1] C. Aluganti Narasimhulu, et al., Atherosclerosis - do we know enough already to prevent it? Curr. Opin. Pharmacol. 27 (2016) 92-102.

[2] T.J. Peters, C. De Duve, Lysosomes of the arterial wall. II. Subcellular fractionation of aortic cells from rabbits with experimental atheroma, Exp. Mol. Pathol. 20 (2) (1974) 228-256.

[3] G. Schmitz, M. Grandl, Endolysosomal phospholipidosis and cytosolic lipid droplet storage and release in macrophages, Biochim. Biophys. Acta 1791 (6) (2009) 524-539.

[4] W.G. Jerome, Lysosomes, cholesterol and atherosclerosis, Clin. Lipidol. 5 (6) (2010) 853-865.

[5] M.S. Brown, J.L. Goldstein, Lipoprotein metabolism in the macrophage: implications for cholesterol deposition in atherosclerosis, Annu. Rev. Biochem. 52 (1983) 223-261.

[6] D. Steinberg, The LDL modification hypothesis of atherogenesis: an update, J. Lipid Res. 50 (Suppl) (2009) S376-S381.

[7] D. Steinberg, J.L. Witztum, Oxidized low-density lipoprotein and atherosclerosis, Arterioscler. Thromb. Vasc. Biol. 30 (12) (2010) 2311-2316.

[8] R. Stocker, J.F. Keaney Jr., Role of oxidative modifications in atherosclerosis, Physiol. Rev. 84 (4) (2004) 1381-1478.

[9] J.A. Berliner, N. Leitinger, S. Tsimikas, The role of oxidized phospholipids in atherosclerosis, J. Lipid Res. 50 (Suppl) (2009) S207-S212.
[10] J.W. Heinecke, Mechanisms of oxidative damage of low density lipoprotein in human atherosclerosis, Curr. Opin. Lipidol. 8 (5) (1997) 268-274.

[11] X. Jiang, et al., Oxidized low density lipoproteins-do we know enough about them? Cardiovasc. Drugs Ther. 25 (5) (2011) 367-377.

[12] S.H. Choi, et al., Lipoprotein accumulation in macrophages via toll-like receptor-4dependent fluid phase uptake, Circ. Res. 104 (12) (2009) 1355-1363.

[13] P.M. Hutchins, E.E. Moore, R.C. Murphy, Electrospray MS/MS reveals extensive and nonspecific oxidation of cholesterol esters in human peripheral vascular lesions, J. Lipid Res. 52 (11) (2011) 2070-2083.

[14] H. Kamido, et al., Lipid ester-bound aldehydes among copper-catalyzed peroxidation products of human plasma lipoproteins, J. Lipid Res. 36 (9) (1995) 1876-1886.

[15] E.A. Podrez, et al., A novel family of atherogenic oxidized phospholipids promotes macrophage foam cell formation via the scavenger receptor CD36 and is enriched in atherosclerotic lesions, J. Biol. Chem. 277 (41) (2002) 38517-38523.

[16] R.G. Salomon, Structural identification and cardiovascular activities of oxidized phospholipids, Circ. Res. 111 (7) (2012) 930-946.

[17] W. Luu, et al., Oxysterols: old tale, new twists, Annu. Rev. Pharmacol. Toxicol. 56 (2016) 447-467.

[18] L.M. Estronca, et al., Molecular etiology of atherogenesis-in vitro induction of lipidosis in macrophages with a new LDL model, PLoS One 7 (4) (2012), e34822.

[19] C. de Duve, The participation of lysosomes in the transformation of smooth muscle cells to foamy cells in the aorta of cholesterol-fed rabbits, Acta Cardiol. (Suppl. 20) (1974) 9-25.

[20] O.V. Vieira, et al., Rapid isolation of low density lipoproteins in a concentrated fraction free from water-soluble plasma antioxidants, J. Lipid Res. 37 (12) (1996) 2715-2721.

[21] K. Stoletov, et al., Vascular lipid accumulation, lipoprotein oxidation, and macrophage lipid uptake in hypercholesterolemic zebrafish, Circ. Res. 104 (8) (2009) 952-960.

[22] A.S. Inacio, et al., In vitro surfactant structure-toxicity relationships: implications for surfactant use in sexually transmitted infection prophylaxis and contraception, PLoS One 6 (5) (2011), e19850.

[23] V.F.D. Donfack, S. R., G. Trigo, P.V.T. Fokou, L.R.Y. Tchokouaha, N. Tsabang, P.H.A Zollo, M.C. Neves, F.F. Boyom, Antimycobacterial activity of selected medicinal plant extracts from Cameroon, Int. J. Biol. Chem. Sci. 8 (1) (2014) 273-288.

[24] A.W. Norman, et al., Studies on the biological properties of polyene antibiotics. Evidence for the direct interaction of filipin with cholesterol, J. Biol. Chem. 247 (6) (1972) 1918-1929.

[25] S.H. Kim, et al., Multi-organ abnormalities and mTORC1 activation in zebrafish model of multiple acyl-CoA dehydrogenase deficiency, PLoS Genet. 9 (6) (2013), e1003563.

[26] C.M. Cardoso, L. Jordao, O.V. Vieira, Rab10 regulates phagosome maturation and its overexpression rescues Mycobacterium-containing phagosomes maturation, Traffic 11 (2) (2010) 221-235.

[27] A.S. Inacio, et al., Quaternary ammonium surfactant structure determines selective toxicity towards bacteria: mechanisms of action and clinical implications in antibacterial prophylaxis, J. Antimicrob. Chemother. 71 (3) (2016) 641-654.

[28] A. Choudhury, et al., Rab proteins mediate Golgi transport of caveola-internalized glycosphingolipids and correct lipid trafficking in Niemann-Pick C cells, J. Clin. Invest. 109 (12) (2002) 1541-1550.

[29] D.K. Sharma, et al., Glycosphingolipids internalized via caveolar-related endocytosis rapidly merge with the clathrin pathway in early endosomes and form microdomains for recycling, J. Biol. Chem. 278 (9) (2003) 7564-7572.

[30] J.A. Mindell, Lysosomal acidification mechanisms, Annu. Rev. Physiol. 74 (2012) 69-86.

[31] F. Schroeder, J.F. Holland, L.L. Bieber, Fluorometric investigations of the interaction of polyene antibiotics with sterols, Biochemistry 11 (16) (1972) 3105-3111.

[32] R. Emanuel, et al., Induction of lysosomal biogenesis in atherosclerotic macrophages can rescue lipid-induced lysosomal dysfunction and downstream sequelae, Arterioscler. Thromb. Vasc. Biol. 34 (9) (2014) 1942-1952.

[33] P. Libby, G.K. Hansson, Inflammation and immunity in diseases of the arterial tree: players and layers, Circ. Res. 116 (2) (2015) 307-311

[34] P. Libby, P.M. Ridker, G.K. Hansson, Inflammation in atherosclerosis: from pathophysiology to practice, J. Am. Coll. Cardiol. 54 (23) (2009) 2129-2138.

[35] G.K. Hansson, P. Libby, I. Tabas, Inflammation and plaque vulnerability, J. Intern. Med. 278 (5) (2015) 483-493.

[36] L. Fang, et al., In vivo visualization and attenuation of oxidized lipid accumulation in hypercholesterolemic zebrafish, J. Clin. Invest. 121 (12) (2011) 4861-4869.

[37] K. Kobayashi, et al., A specific ligand for beta(2)-glycoprotein I mediates autoantibody-dependent uptake of oxidized low density lipoprotein by macrophages, J. Lipid Res. 42 (5) (2001) 697-709.

[38] K. Kobayashi, et al., Circulating oxidized LDL forms complexes with beta2-glycoprotein I: implication as an atherogenic autoantigen, J. Lipid Res. 44 (4) (2003) 716-726.

[39] T.P. Greco, et al., Oxidized-LDL/beta(2)-glycoprotein I complexes are associated with disease severity and increased risk for adverse outcomes in patients with acute coronary syndromes, Am. J. Clin. Pathol. 133 (5) (2010) 737-743.

[40] B.E. Cox, et al., Effects of cellular cholesterol loading on macrophage foam cell lysosome acidification, J. Lipid Res. 48 (5) (2007) 1012-1021.

[41] K.J. Moore, I. Tabas, Macrophages in the pathogenesis of atherosclerosis, Cell 145 (3) (2011) 341-355.

[42] A. Tedgui, Z. Mallat, Cytokines in atherosclerosis: pathogenic and regulatory pathways, Physiol. Rev. 86 (2) (2006) 515-581.

[43] F. Progatzky, et al., Dietary cholesterol directly induces acute inflammasomedependent intestinal inflammation, Nat. Commun. 5 (2014) 5864.

[44] M. van der Vaart, H.P. Spaink, A.H. Meijer, Pathogen recognition and activation of the innate immune response in zebrafish, Adv. Hematol. 2012 (2012) 159807.

[45] C. Stein, et al., Conservation and divergence of gene families encoding components of innate immune response systems in zebrafish, Genome Biol. 8 (11) (2007) R251. 